\title{
L'appel à un laboratoire en sciences sociales par une agence
}

\section{Le cas de l'agence Publika}

David Gracia, chef de projet webmarketing en poste lors de l'étude

Agence Publika

Alexandre Coutant, Professeur

Université de Québec à Montréal

coutant.alexandre@gmail. 
Cette contribution rend compte de la démarche d'appel à un laboratoire de sciences sociales par l'agence Publika. David Gracia, Chef de projet webmarketing en poste au moment de la réalisation de l'étude, décrit le type d'intervention fourni auprès de leurs clients à travers ce cas, afin d'identifier les situations où une approche académique leur est apparue nécessaire. Alexandre Coutant, responsable des interventions du laboratoire, ajoute ses commentaires, identifiés en italique, aux passages qui lui apparaissent charnières dans l'établissement d'une relation mutuellement profitable.

\section{Présentation du client, de son besoin et de la prestation initiale confiée à Publika}

\subsection{Typologie des clients et de leur besoin}

Nous travaillons principalement avec des PME de tous secteurs. Il s'agit majoritairement de PME bien installées qui souhaitent franchir un nouveau palier de développement, soit en accroissant leurs parts de marché, soit en étendant leur zone de chalandise. Internet représente souvent dans leur esprit la solution idoine pour remplir ces objectifs : il leur permet de limiter les coûts (comparé à d'autres supports) et de mieux mesurer leur retour sur investissement.

$\mathrm{Du}$ fait de notre implantation historique dans le Sud de la France, nous avons de nombreuses agences immobilières pour client. Leurs demandes sont plutôt classiques : elles souhaitent un beau site et une bonne visibilité (entendre par là un nombre quotidien de contacts, et une présence dans Google sur les mots-clés les plus stratégiques pour leur activité). Rien qui implique à priori de faire appel aux compétences d'un chercheur. C'est pourtant bien une agence immobilière qui a lancé notre première collaboration avec l'équipe du laboratoire ELLIADD.

\subsection{Présentation du client cible et de ses problématiques}

L'entreprise est située sur Marseille : il s'agit d'une entreprise familiale dirigée par un couple. Cette agence dispose déjà d'un site Internet qu'elle utilise à la fois comme levier commercial mais aussi comme un outil de gestion, grâce à de nombreuses fonctionnalités présente en Intranet. Elle dispose également d'un réseau de mini-sites secondaires.

Le PDG a une forte appétence pour les nouvelles technologies et Internet en particulier. Il est persuadé que le développement de son entreprise, sa différenciation sur le marché, passent par une démarche forte autour du numérique. Il a mené l'orientation stratégique au niveau webmarketing jusqu'à présent mais fait appel à nous car il pense avoir atteint ses limites. Il est donc important de préciser que ce 
client dispose de certaines connaissances tant en web qu'en web marketing et une vrai curiosité dans ce domaine. C'est, il me semble, l'une des raisons qui rendent possible cette première collaboration.

Notre prestation avait pour but dans un premier temps de développer la visibilité du site principal sur le moteur de recherche Google. Pour cela nous avons travaillé à une optimisation du code source et du contenu du site, ainsi qu'à une meilleure liaison entre les différents sites de son réseau. Tout ceci dans le but de maximiser la synergie entre les sites au niveau du positionnement Google. Nous avons par la suite lancé une campagne de liens externes qui consiste à obtenir un certain nombre de liens hypertextes sur des sites externes, mais de thématiques proches, pointant vers le site du client. La hausse de visibilité enregistrée suite à ces différentes actions a engendré de nouveaux questionnements. Bien que déjà abordée, une autre problématique est apparue plus prioritaire aux yeux du client: que font les internautes une fois arrivés sur son site?

En effet, si le client a constaté l'évolution positive de sa présence sur le moteur de recherche Google, il a également remarqué comme nous, que le nombre de contacts ne suivait pas la même courbe. La problématique s'est alors recentrée sur des problématiques navigationnelles sous-tendant des objectifs, en terme marketing, de conversion.

\section{Contexte technique de l'étude}

\subsection{Une approche quantitative et professionnalisée}

L'aspect navigation est rapidement venu au centre des débats car, comme nous l'avions évoqué précédemment, le client dispose de son site Internet depuis plusieurs années. En tant qu'agence de communication, nous assurons à la fois des prestations de référencement naturel mais également de la création de site et de la création graphique. Lorsque des problématiques de « conversion » apparaissent, nous tentons de les résoudre en travaillant sur deux axes :

- Nous analysons d'une part les statistiques de navigation en axant nos travaux sur les données issus de Google Analytics. Nous travaillons autour d'indicateurs repères comme le temps moyen passé sur le site, le taux de rebond, le nombre de pages par visite... Nous interprétons ces valeurs au prisme de différents filtres comme les principaux chemins de navigation, l'origine des internautes (type de source de trafic apportant la visite), les mots clés, etc.

- Nous associons ces analyses statistiques à un audit graphique. Nous faisons intervenir un webdesigner afin qu'il apporte son regard, et juge les interfaces qui lui sont présentées. Il s'agit de savoir si les interfaces du site sont fonctionnelles, toujours en phase avec leur époque. 
Cette approche permet de dégager des pistes d'optimisation. Les analyses statistiques révèlent à quel moment l'internaute abandonne sa démarche, quelles sont les zones perfectibles ou bloquantes. L'audit graphique souligne les carences visuelles, contextualise ces difficultés.

\subsection{Perspectives nouvelles}

Ces travaux ne satisfont pas totalement le client. L'analyse des statistiques amène souvent beaucoup de conjectures et pose de nouvelles questions : qu'est ce qui fait fuir/bloque les internautes, pourquoi la page de contact est si peu visitée, etc. sans que l'on puisse répondre de façon certaine. L'audit graphique est avant tout centré sur un ensemble de « bonnes pratiques».

Ces dernières sont diffusées par une communauté professionnelle qui s'entend autour de principes d'organisation esthétique et logique qui font consensus dans un contexte donné. Elles font souvent écho aux tendances, aux modes. Elles peinent parfois à pointer les carences propres à un site, en distillant des recommandations peut être trop générales pour ce type de problématiques.

Si l'ensemble de la démarche a su apporter une évolution positive, nous n'arrivions pas totalement à répondre à la problématique du client qui souhaitait une approche moins généralisante, qui puisse apporter une analyse navigationnelle plus centrée sur son site. Il avait besoin d'un audit approfondi sur la navigabilité.

C'est à ce moment que nous choisissons de faire appel aux compétences des chercheurs d'ELLIADD.

C'est dans ce type de contexte que l'intervention d'un chercheur spécialiste des usages prend tout son intérêt. La méthodologie de travail suivie par Publika relève en effet d'habitudes professionnelles éprouvées pour des activités d'internautes proches des «usagers représentés» présents dans l'esprit des concepteurs spécialisés dans le web. Si le gain en termes de productivité est évident tant que les usagers réels ne s'en écartent pas sensiblement, elles se révéleront en revanche peu aptes à rendre compte de situations où les logiques de ces derniers paraissent échapper à notre compréhension.

En effet, les outils de Google Analytics fournissent certes une quantification dont on sait le pouvoir attractif dans les métiers de la gestion, le webmarketing ne faisant pas exception - mais de critères qui demeurent

1. Génériques. Google Analytics produit ses mesures en fonction d'une définition canonique de la performance qui n'épouse pas nécessairement la diversité des objectifs que fixent les organisations à leur présence en ligne - nous renvoyons 
ici à l'analyse détaillée de l'esprit ${ }^{150}$ de cet outil qu'effectue Thomas Grignon dans ce numéro

2. En attente d'interprétation. Les activités quantifiées par Google Analytics s'inscrivent généralement dans une pratique plus large (consommation, recherche d'information, sociabilité, échanges d'avis entre pairs, etc.), multisupports, ancrée socialement, spatialement et temporellement. En décortiquant ces dernières et en n'en retenant que le volet mesurable en ligne, le logiciel échoue à retranscrire le sens de ces pratiques. S'il produit de nombreux chiffres, il n'aide en rien à leur interprétation en dehors de son esprit très particulier évoqué précédemment. Employé sans être intégré dans une démarche méthodologique autonome, il amène à de nombreux contresens d'autant plus difficiles à identifier que ce sera encore Google Analytics qui sera employé pour tenter de répondre aux insatisfactions du commanditaire.

Par ailleurs, l'expertise d'un webdesigner de métier se fonde usuellement sur une expérience personnelle associée à des règles générales de design. Si cette expérience et cette expertise suffisent ici aussi la plupart du temps à produire des dispositifs adaptés, les nombreux résultats de la sociologie de l'innovation illustrent comment elles peuvent au contraire occulter la prise en compte des usagers effectifs dès lors que ceux-ci s'écartent de ce que le designer juge logique et cohérent ${ }^{151}$.

Ajoutons, en termes de possibilité de dialogue, que ce moment de doute face à la difficulté à comprendre l'internaute ciblé constitue un moment clé pour envisager ce type de collaboration. L'utilité d'un chercheur en sciences sociales n'est pas nécessairement évidente parmi les praticiens. Cela est d'autant plus vrai dans les secteurs du marketing et de la communication, qui produisent endogènement nombre d'analystes et de penseurs de la profession. L'intervention au moment où les praticiens ont conscience des limites de leurs méthodes usuelles permet donc d'ouvrir ces derniers à la possibilité d'une démarche alternative. Par ailleurs, proposer de comprendre une situation complexe, incertaine pour les acteurs impliqués, constitue un cas intéressant pour les chercheurs, dépassant la seule

\footnotetext{
${ }^{150}$ Nous employons esprit dans la lignée de ce qu'ont défini Desanctis et Poole (1994) à propos des technologies, c'est-à-dire la finalité pour laquelle celles-ci ont été développées, qui oriente et transparaît dans son design comme dans ses fonctionnalités.

${ }^{151}$ Il n'est pas lieu ici de développer une analyse critique des outils censés replacer l'usager au cœur de la démarche du concepteur en informatique et en ergonomie, comme cela a été effectué à propos du marketing (Cochoy, 1999). Des résultats d'enquête concluant au même éloignement entre l'intention et la pratique effective des concepteurs s'accumulent cependant en mettant en lumière les mêmes multiples raisons idéologiques, organisationnelles ou économiques. Le lecteur intéressé pourra trouver des exemples plus développés dans le récent numéro de la revue Communication «Les publics imaginés et réels des professionnels d'Internet»: http://communication.revues.org/
} 
analyse de la diffusion régulièrement dénoncée (Jouët, 2011). Il ne s'agit pas de se plier à une demande sociale de mesure de critères non problématisés. Il s'agit bien de tester la valeur explicative de méthodologies et de théories focalisées sur les usagers pris en contexte, peu répandues hors laboratoires de recherche ${ }^{152}$. L'utilité sociale de la recherche s'en trouve aussi renforcée puisque la démonstration de l'efficacité d'une approche respectant le contexte d'activité de l'usager contribue à encourager des méthodes de travail intégrant une vision plus éthique des relations entre des organisations et leurs publics.

Les objectifs de cette collaboration étaient multiples :

- Apporter un regard neuf et extérieur qui puisse orienter les analyses sur de nouveaux aspects. Il s'agissait de sortir du regard purement professionnel, empli de praxis, et de le confronter avec des usages.

- Proposer une méthodologie renouvelée et approfondir les questionnements.

- Assurer un transfert de compétence nous permettant à terme de mobiliser ce savoir-faire.

Montrer au client notre volonté de répondre au mieux à ces besoins en cherchant à l'extérieur les compétences nécessaires.

La position d'extériorité des chercheurs impliqués s'avère effectivement essentielle. Elle constitue bien entendu un argument commercial pour l'agence, qui justifie ainsi d'une méthode inusitée par ses concurrentes. Cependant, elle est aussi une condition de l'établissement d'une relation de confiance originale, qui s'éloigne de la seule relation commerciale. Si le laboratoire est rétribué, cette rétribution ne sert qu'indirectement les chercheurs dans le cadre de leurs activités de recherche (soutien à la publication, déplacements pour effectuer des terrains ou assister à des manifestations scientifiques). Par ailleurs, elle n'est aucunement liée à leur salaire et demeure donc facultative par rapport à l'activité d'un consultant vivant de ses mandats. L'intérêt majeur, pour ces derniers, demeure donc la possibilité d'éprouver la valeur explicative et de guide pour l'action d'approches théoriques. Le commanditaire peut donc d'autant plus être en confiance qu'il s'associe à des prestataires guidés essentiellement par l'envie de comprendre son problème et d'y apporter une solution.

Dans une perspective d'amélioration des relations entre organisations et publics permise par l'intervention des chercheurs en sciences sociales au sein de la société qu'ils analysent, le transfert de compétence s'avérera cependant essentiel. Il pérennisera effectivement cette conception respectueuse de l'usager en contexte

\footnotetext{
${ }^{152}$ Notons l'exception très stimulante de la méthode CAUTIC formulée dans les années 90 et ayant récemment guidé l'ouvrage Innover par les usages (Pizelle, Hoffmann, Verchère, Aubouy, 2014)
} 
dans les routines de l'agence. Ajoutons que dans un contexte de difficile reconnaissance des compétences communicationnelles, celle-ci nous semble essentielle pour que le mandat des agences spécialisées dans le web ne soit pas limité à la réalisation technique mais qu'on leur reconnaisse une véritable compétence stratégique, essentielle à la qualité du développement d'une présence en ligne.

\section{Temporalités et relations commerciales}

S'il y a un aspect inévitable à aborder lorsque l'on parle d'une collaboration entreprise/laboratoire public, c'est celui de la temporalité.

En effet, le temps «économique», celui des affaires commerciales, des campagnes marketing est un temps court, caractérisé par des périodes pleines, souvent stratégiques pour l'activité de l'entreprise et sur lesquelles on ne peut faire l'impasse. Les plannings sont donc souvent très courts et les délais entre la prise de décision et la mise en place d'une action à l'issue de la décision en question sont souvent très réduits, marqués par une certaine immédiateté. Ce temps court, où le présent est surinvesti peut parfois se heurter au temps de l'analyse, de la recherche universitaire où la prise de décision est plus longue et les délais plus importants.

La collaboration entre entreprise et laboratoire doit donc prendre en considération dès les premiers échanges, ces différences de temporalité et aborder la question du planning. La capacité de l'entreprise à accepter un délai de livraison raisonnable pour le déroulement des travaux de recherche dépend souvent des échéances économiques en rapport avec son besoin. La question de la faisabilité temporelle doit donc être posée assez rapidement.

Dans le cadre de cette collaboration tripartite, la difficulté est elle aussi multipliée par trois : il faut répondre à la fois à la temporalité du client, celle de notre prestation qui ne doit pas prendre de retard, et trouver un délai raisonnable pour mener des expérimentations qui apporteront des résultats concluants. Nous travaillons donc ensemble pour définir un planning de livraison et prévoir des plages de travail pour mettre en place les modifications éventuelles, qui découleront des travaux de recherche. Il me semble que ce travail préparatoire est essentiel à la bonne réception des opérations de recherche et à la mise en place des recommandations qui en découle.

L'exercice est très instructif pour les chercheurs s'intéressant à l'innovation et aux méthodes de travail en marketing et communication. Ces contraintes temporelles, auxquelles il convient parfois d'associer d'autres plus organisationnelles ou économiques, amènent souvent à nuancer les théories mobilisées. Un accompagnement direct permet de constater les effets de dépendances de ces différentes contraintes, qui peuvent amener à ce que des problèmes identifiés demeurent ignorés car ils remettraient en cause tout l'existant. L'interrogation sur la capacité des théories des sciences sociales à 
comprendre mais aussi à guider l'action ${ }^{153} \mathrm{~s}$ 'en trouve stimulée. Elle constitue une invitation à la prise en compte toujours plus grande de la complexité, et bien entendu à de l'humilité dans la présentation des résultats des enquêtes.

Sur un plan pratique, le transfert des méthodologies scientifiques vers des méthodes de travail employables par des praticiens avec leurs contraintes professionnelles nécessite aussi un approfondissement. Il est poursuivi selon deux angles principaux :

- Le premier consiste à identifier tous les moyens méthodologiques permettant d'observer et/ou interroger les usagers (par exemple à travers les outils de l'UX design);

- Le second consiste à jauger à quel moment la réduction du temps consacré à la prise en compte des usagers aboutit à la reproduction des visions des usagers des concepteurs.

Il faut également parfois jouer les interprètes et assurer que tout le monde parle le même langage, que les termes employés sont compris de tous. En ce sens il est nécessaire que le client dispose d'un minimum de culture web pour comprendre la méthodologie et l'intérêt global de la prestation

\section{Apport de l'étude au client}

L'étude s'est présentée sous la forme d'un audit de navigabilité. Ce document a été livré après une première lecture de l'équipe webmarketing de Publika. Il s'agissait pour l'équipe de l'agence d'anticiper sur les questions et réflexions éventuelles du client.

\subsection{La réception de l'étude}

Le client a donc pris connaissance du document via une lecture en solitaire sans éclaircissement de notre part ou du laboratoire. Le but étant qu'il se forge d'abord sa propre opinion et ses propres idées. Dans un deuxième temps le client a demandé un échange au responsable webmarketing de Publika autour de l'audit, afin de répondre à un ensemble de questions.

Le client a ainsi manifesté le besoin d'un éclairage de notre part et ce pour plusieurs raisons :

\footnotetext{
${ }^{153}$ Rappelons que Durkheim considérait que « nous estimerions que nos recherches ne méritent pas une heure de peine si elles ne devaient avoir qu'un intérêt spéculatif. Si nous séparons avec soin les problèmes théoriques des problèmes pratiques, ce n'est pas pour négliger ces derniers : c'est, au contraire, pour nous mettre en état de les mieux résoudre » (1893, p. XXXIX)
} 
- Il avait besoin de notre expertise pour valider sa compréhension de l'audit. En d'autres termes : est-ce que j'ai bien compris ce que j'ai lu.

- Il souhaitait avoir notre point de vue sur l'audit et les conclusions de ce dernier.

- Il souhaitait enfin avoir un rapide retour concernant les coûts qu'impliquaient les modifications techniques préconisées par l'audit.

À la suite de cet entretien, le client a demandé également qu'un point téléphonique soit réalisé avec le chercheur responsable de l'étude afin d'avoir également son éclairage sur différents points. Cet entretien lui a permis de disposer de suffisamment de réponses pour nourrir son processus de décisions.

Pour le chercheur, la question de la compétence de traduction de son expertise en recommandations tout autant compréhensibles par le commanditaire que respectueuses de ses analyses, est cruciale. Il nous apparaît qu'elle vient avec l'expérience, les questions portant sur la compréhension de l'audit se faisant plus rares à mesure des collaborations. Néanmoins, celle-ci ne remplace pas une démarche initiale de la part du chercheur d'assimilation des éléments de conception professionnels, afin de les intégrer à sa pratique: logiciels de mockups, modèles d'audits, benchmarking, etc. Si l'expérience est très riche pour comprendre les esprits intégrés aux outils de travail employés dans les secteurs du marketing et de la communication, elle nécessite une ouverture à des univers s'écartant du canon des lectures, méthodes et théories usuellement fréquentées dans le quotidien universitaire. Il ne s'agit cependant aucunement de passer de l'un à l'autre ou de soumettre la démarche scientifique à ces modes de travail mais bien au contraire d'intégrer ces éléments de conception au sein d'une démarche rigoureuse telle que celles mises en place dans les enquêtes en sciences sociales afin de les employer de manière réflexive. La question n'est donc en rien selon nous de savoir si des chercheurs peuvent effectuer du travail de consultation, mais à l'inverse de voir si intégrer les modes de travail du consulting dans une démarche plus large de rigueur scientifique augmente leurs valeurs explicatives et de guide pour l'action.

\section{Bibliographie}

Desanctis G., Poole MS. (1994), « Capturing the complexity in advanced technology use: adaptative structuration theory », Organization science, vol. 5, n², pp 121146.

Durkheim É. (1893), De la division du travail social, Paris, PUF.

Jouët J. (2011), « Des usages de la télématique aux Internet Studies » in Julie Denouël \& Fabien Granjon, dir., Communiquer à l'ère numérique. Regards croisés sur la sociologie des usages, Paris, Presses des Mines, p. 45-90.

Pizelle P., Hoffmann J., Verchère C., Aubouy M. (2014), Innover par les usages, Grenoble, Editions d'Innovations. 